\title{
Decoherence in a superconducting flux qubit with a $\pi$-junction
}

\author{
T. Kato, ${ }^{1}$ A. A. Golubov, ${ }^{2}$ and Y. Nakamura ${ }^{3,4}$ \\ ${ }^{1}$ Institute for Solid State Physics, The University of Tokyo, Kashiwa, Chiba 277-8581, Japan \\ ${ }^{2}$ Faculty of Science and Technology, University of Twente, 7500 AE Enschede, The Netherlands \\ ${ }^{3}$ Nano Electronics Research Laboratories, NEC Corporation, Tsukuba, Ibaraki 305-8501, Japan \\ ${ }^{4}$ Frontier Research System, The Institute of Physical and Chemical Research (RIKEN), Wako, Saitama 351-0198, Japan
}

(Received 20 August 2007; revised manuscript received 1 October 2007; published 15 November 2007)

\begin{abstract}
We consider the use of a $\pi$ junction for flux qubits to realize degenerate quantum levels without external magnetic field. On the basis of the Caldeira-Leggett model, we derive an effective spin-Boson model and study decoherence of this type of qubits. We estimate the dephasing time by using parameters from recent experiments of superconductor-insulator-ferromagnet-superconductor junctions and show that high critical current and large subgap resistance are required for the $\pi$ junction to realize a long coherent time.
\end{abstract}

DOI: $10.1103 /$ PhysRevB.76.172502

PACS number(s): 74.90.+n, 03.67.Lx, 03.65.Yz

It is now well established that, in addition to conventional Josephson junctions having an energy minimum at zero phase difference across the junction, there exist the so-called $\pi$ junctions which provide the phase shift of $\pi$ in the ground state. The intrinsic $\pi$ shifts were first realized in grain boundary Josephson junctions in $d$-wave superconductors. ${ }^{1,2}$ Subsequently, $\pi$ junctions have been realized in hybrid structures between high- $T_{\mathrm{c}}$ and low- $T_{\mathrm{c}}$ superconductors ${ }^{2,3}$ and by injection of quasiparticles. ${ }^{4}$ Recent development in fabrication of superconductor-ferromagnet-superconductor (SFS) junction made it possible to obtain a $\pi$ junction with high critical current density. ${ }^{5}$ An advantage of SFS junctions is the possibility to combine them with usual low- $T_{\mathrm{c}}$ superconductive circuits using conventional fabrication technique.

The use of $\pi$ junctions provides several applications. For example, the application of $\pi$ junctions as complementary devices in single flux quantum logic was recently proposed ${ }^{6}$ and realized in high- $T_{\mathrm{c}}$-low- $T_{\mathrm{c}}$ junctions. ${ }^{7}$ It is interesting that before this "classical" application of the $\pi$ junction, the use of $\pi$ junctions for realization of quantum two-state systems was considered. ${ }^{8}$ In this qubit system, the $\pi$ junction was used as a $\pi$ phase shifter along the loop instead of current biasing or external magnetic flux. After this proposal, remarkable progress in fabrication, coherent control of one qubit, controllable coupling between qubits, and readout with high fidelity has been achieved in superconducting qubits. $^{9-13}$ Nevertheless, up to now, the use of $\pi$ junctions to qubits has not been studied experimentally. One of the difficulties for realization may lie on the original proposal in which a qubit consists of complicated circuits with many Josephson junctions. ${ }^{8}$ Another serious difficulty comes from dissipation due to quasiparticle excitation, which is unavoidable in many realizations of $\pi$ junctions. Generally, qubits suffer strong decoherence by excitation in the environment. ${ }^{14-16}$

In this Brief Report, we consider the use of $\pi$ junction for phase bias of flux qubits. The circuit we study is shown in Fig. 1. In this circuit, we need no external flux to realize degenerate quantum levels because the phase drop across the three Josephson junctions is adjusted as $\pi$ by the $\pi$ junction with a large Josephson energy. This type of phase bias can avoid dephasing due to noise in external flux and is frequently called as a "quiet qubit." In actual experiments, how- ever, damping at the $\pi$ junction may cause severe decoherence on the qubit. The purpose of this Brief Report is to derive the effective spin-Boson model describing the flux qubit with a damped $\pi$ junction and to estimate the dephasing time by using realistic experimental parameters. We clarify the condition for long coherence time in this qubit system and discuss the possibility of the use of $\pi$ junctions for qubits by referring recent experiments on superconductor-insulator-ferromagnet-superconductor (SIFS) junctions.

In order to describe damped dynamics, we introduce the resistively shunted junction (RSJ) model for the $\pi$ junction, as shown in Fig. 1, where dissipation is expressed by a resistance $R$ shunted in parallel to the junction. We expect that this phenomenological model may give a qualitative estimate of decoherence effects by $\pi$ junctions. We introduce the charging energy $E_{C, \pi}=e^{2} /\left(2 C_{\pi}\right)$ and damping frequency $\gamma$ $=1 /\left(R C_{\pi}\right)$ of the $\pi$ junction.

The Hamiltonian consists of three parts as $H=H_{\text {qubit }}+H_{L}$ $+H_{\pi}$. The first part $H_{\text {qubit }}$ describes a flux qubit and is given as $^{12}$

$$
\begin{aligned}
H_{\text {qubit }}= & -E_{J}\left(\cos \phi_{1}+\cos \phi_{2}+\alpha \cos \phi_{3}\right) \\
& +4 E_{C}\left(n_{1}^{2}+n_{2}^{2}+\alpha^{-1} n_{3}^{2}\right),
\end{aligned}
$$

where $E_{J}$ is a Josephson energy and $E_{C}$ is a charging energy. Here, $\phi_{i}$ and $n_{i}$ are a phase difference and induced charge at the $i$ th junction, respectively. The area of one junction is reduced by the factor $\alpha$, which is typically taken as $0.8 .^{12}$

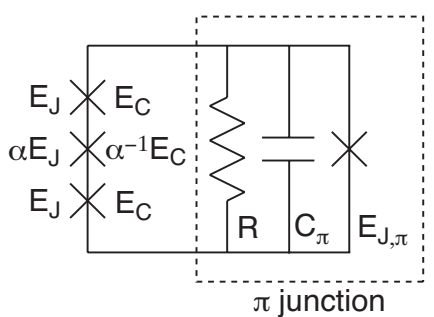

FIG. 1. Flux qubit circuits with a $\pi$ junction. Shunt resistance at the $\pi$ junction is introduced for estimate of damping effects. 
The second part of the Hamiltonian describes the inductance energy of the loop and is given as

$$
H_{L}=\frac{1}{2 L}\left(\frac{\Phi_{0}}{2 \pi}\right)^{2}\left(\phi_{1}+\phi_{2}+\phi_{3}+\phi_{\pi}-\phi_{\mathrm{ext}}\right)^{2},
$$

where $\Phi_{0}=h /(2 e)$. Here, $\phi_{\pi}$ is a phase of the $\pi$ junction, and $\phi_{\mathrm{ext}}=2 \pi\left(\Phi_{\mathrm{ext}} / \Phi_{0}\right)$ is a phase induced by the external flux through the loop. By assuming small inductance $L$, the inductive part of the Hamiltonian can be treated as a constraint condition

$$
\phi_{1}+\phi_{2}+\phi_{3}+\phi_{\pi}=\phi_{\mathrm{ext}} .
$$

The third term describes the damped $\pi$ junctions and is expressed by the Caldeira-Leggett Hamiltonian,

$$
\begin{aligned}
H_{\pi}= & +E_{J, \pi} \cos \phi_{\pi}+4 E_{C, \pi} n_{\pi}^{2} \\
& +\sum_{\alpha}\left[\frac{p_{\alpha}^{2}}{2 m_{\alpha}}+\frac{1}{2} m_{\alpha} \omega_{\alpha}^{2}\left(x_{\alpha}-\frac{C_{\alpha}}{m_{\alpha} \omega_{\alpha}^{2}} \phi_{\pi}\right)^{2}\right] .
\end{aligned}
$$

The damping property is determined by the spectral function,

$$
J(\omega)=\frac{\pi}{2} \sum_{\alpha} \frac{C_{\alpha}^{2}}{m_{\alpha} \omega_{\alpha}} \delta\left(\omega-\omega_{\alpha}\right) .
$$

In the RSJ model, the spectral function is given as

$$
J(\omega) / M=\gamma \omega e^{-\omega / \omega_{c}},
$$

where $M=1 /\left(8 E_{C, \pi}\right)$ is a mass of the $\pi$ junction and $\omega_{c}$ is a high-frequency cutoff.

In this Brief Report, we focus on the "passive" use of the $\pi$ junction. For this use, the Josephson energy of the $\pi$ junction should be taken as sufficiently large. Hence, we assume $E_{J, \pi} \gg E_{C}, E_{J}$ and approximate the Josephson energy of the $\pi$ junction as $E_{J, \pi}\left(\phi_{\pi}-\pi\right)^{2} / 2$. Within this approximation, the phase of the $\pi$ junction is kept almost $\pi$. The remaining dynamics around the potential minima is described by a damped oscillator with a eigenfrequency $\omega_{0}$ $=\left(8 E_{J, \pi} E_{C, \pi}\right)^{1 / 2} / \hbar$. In the following discussion, we set $\hbar=1$.

Under the condition $E_{J} \gg E_{C}$, which is taken for usual flux qubits, we can truncate the Hamiltonian $H_{\text {qubit }}$ into the twolevel Hamiltonian as $H_{\text {qubit }}=H_{\text {two-state }}+H_{\text {coupling. }}$. The first part $H_{\text {two-state }}=(\Delta / 2) \sigma_{x}+(\varepsilon / 2) \sigma_{z}$ describes the qubit system, where $\Delta$ is a tunneling splitting and $\varepsilon$ is a bias proportional to the external flux $\Phi_{\text {ext }}$. The second part, which describes the coupling between the qubit and the $\pi$ junction, is given as

$$
H_{\text {coupling }}=-E_{J, \text { eff }}(\alpha) \Delta \phi_{\pi} \sigma_{z},
$$

where $E_{J \text { eff }}=\left[1-1 /\left(4 \alpha^{2}\right)\right]^{1 / 2} E_{J}$ and $\Delta \phi_{\pi}=\phi_{\pi}-\pi$.

To simplify the Hamiltonian $H_{\pi}$, we change the variables as $x=M^{1 / 2} \Delta \phi_{\pi}$ and $p=M^{-1 / 2} n_{\pi}$. We further replace the sum in the Hamiltonian of the harmonic oscillators by the integral. This can be performed by replacing the variables as $X_{\omega}=m^{1 / 2} x_{\alpha} /(\Delta \omega)^{1 / 2}, \quad P_{\omega}=m^{-1 / 2} p_{\alpha} /(\Delta \omega)^{1 / 2}, \quad$ and $\quad C_{\omega}$ $=\left(M m_{\alpha}\right)^{-1 / 2} C_{\alpha} /(\Delta \omega)^{1 / 2}$, where $\Delta \omega$ is a length of one slice in the $\omega$ direction. In the limit $\Delta \omega \rightarrow 0$, we obtain

$$
\begin{aligned}
H_{\pi}= & \frac{\hat{p}^{2}}{2}+\frac{1}{2} \omega_{0}^{2} \hat{x}^{2}+\int_{0}^{\infty} d \omega\left\{\frac{P_{\omega}^{2}}{2}+\frac{1}{2} \omega^{2} X_{\omega}^{2}-C_{\omega} X_{\omega} \hat{x}\right. \\
& \left.+\frac{C_{\omega}}{2 \omega^{2}} \hat{x}^{2}\right\} .
\end{aligned}
$$

The coefficient $C_{\omega}$ can be related to the spectral function as

$$
J(\omega) / M=\frac{\pi}{2} \frac{C_{\omega}^{2}}{\omega} .
$$

The Hamiltonian of the $\pi$ junction describing a damped oscillator can be diagonalized exactly. ${ }^{17,18}$ In order to express the eigenmodes with the energy $\omega$, we introduce a canonical transformation for the operators as

$$
\bar{X}_{\omega}=a(\omega) \hat{x}+\int_{0}^{\infty} d \omega^{\prime} b_{\omega^{\prime}}(\omega) X_{\omega^{\prime}},
$$

where the coefficients $a(\omega)$ and $b_{\omega^{\prime}}(\omega)$ are assumed to be real. The coefficients are chosen to satisfy the eigenmode equations

$$
\bar{\omega}_{0}^{2} a(\omega)+\int_{0}^{\infty} d \omega^{\prime} C_{\omega^{\prime}} b_{\omega^{\prime}}(\omega)=\omega^{2} a(\omega),
$$

$$
C_{\omega^{\prime}} a(\omega)+\omega^{\prime 2} b_{\omega^{\prime}}(\omega)=\omega^{2} b_{\omega^{\prime}}(\omega),
$$

where $\bar{\omega}_{0}^{2}=\omega_{0}^{2}+\int d \omega^{\prime} C_{\omega^{\prime 2}} / \omega^{\prime 2}$. Then, the Hamiltonian of the $\pi$ junction can be diagonalized as

$$
H_{\pi}=\int_{0}^{\infty} d \omega\left(\frac{\bar{P}_{\omega}^{2}}{2}+\frac{1}{2} \omega^{2} \bar{X}_{\omega}^{2}\right) .
$$

In order to solve the eigenmode equations, Eqs. (11) and (12), we may follow the calculation in Fano's paper. ${ }^{17} \mathrm{We}$ only give the result for $a(\omega)$ as

$$
\begin{gathered}
|a(\omega)|^{2}=\frac{C_{\omega}^{2}}{\left(\pi^{2} C_{\omega}^{4} / 4 \omega^{2}\right)+\left[\omega^{2}-\bar{\omega}_{0}^{2}-F(\omega)\right]^{2}}, \\
F(\omega)=P \int_{0}^{\infty} d \omega^{\prime} \frac{C_{\omega^{\prime 2}}}{\left(\omega^{2}-\omega^{\prime 2}\right)} .
\end{gathered}
$$

The part of the energy renormalization is modified as

$$
\bar{\omega}_{0}^{2}+F(\omega)=\omega_{0}^{2}+P \int_{0}^{\infty} d \omega^{\prime} \frac{\omega^{2} C_{\omega^{\prime 2}}}{\omega^{\prime 2}\left(\omega^{2}-\omega^{\prime 2}\right)} .
$$

Here, the second term in right-hand side can be neglected because it can be shown to be $O\left(\Delta / \omega_{c}\right)$.

Thus, we obtain the expression for the $\pi$ junction as Eq. (13), while the coupling term (7) is rewritten by the relation

$$
x=\int_{0}^{\infty} d \omega^{\prime} a\left(\omega^{\prime}\right) \bar{X}_{\omega^{\prime}} .
$$

As a result, we obtain the total Hamiltonian as 


$$
\begin{aligned}
H= & \frac{\Delta}{2} \sigma_{x}+\frac{\varepsilon}{2} \sigma_{z}-E_{J, \mathrm{eff}}\left(8 E_{C, \pi}\right)^{1 / 2} \sigma_{z} \int_{0}^{\infty} d \omega a(\omega) \bar{X}_{\omega} \\
& +\int_{0}^{\infty} d \omega\left(\frac{\bar{P}_{\omega}^{2}}{2}+\frac{1}{2} \omega^{2} \bar{X}_{\omega}^{2}\right) .
\end{aligned}
$$

In this modified spin-Boson model, the effective spectral function is given by

$$
\begin{aligned}
J_{\mathrm{eff}}(\omega) & =8 E_{J, \mathrm{eff}}^{2} E_{C, \pi} \frac{\pi}{2} \frac{|a(\omega)|^{2}}{\omega} \\
& =8 E_{J, \mathrm{eff}}^{2} E_{C, \pi} \frac{\left(\pi C_{\omega}^{2} / 2 \omega\right)}{\left(\pi C_{\omega}^{2} / 2 \omega\right)^{2}+\left(\omega^{2}-\omega_{0}^{2}\right)^{2}} .
\end{aligned}
$$

For the RSJ model, by substituting $J(\omega) / M=\pi C_{\omega}^{2} /(2 \omega)$ $=\gamma \omega$, the effective spectral function is obtained for $\omega \ll \omega_{c}$ as

$$
J_{\mathrm{eff}}(\omega)=8 E_{J, \mathrm{eff}}^{2} E_{C, \pi}\left[\frac{\gamma \omega}{\gamma^{2} \omega^{2}+\left(\omega^{2}-\omega_{0}^{2}\right)^{2}}\right] .
$$

Note that the form factor of the damped oscillator (the factor in the bracket) appears in the effective spectral function.

By using the effective spectral function $J_{\text {eff }}(\omega)$, we estimate the dephasing time of the qubit at the optimal point $(\varepsilon=0)$, where long coherence time is realized by suppressing a linear coupling to the heat bath. The dephasing time is evaluated within the spin-Boson model in the form ${ }^{14,19,20}$

$$
\tau_{\varphi}^{-1}=\frac{1}{2} \tau_{\text {relax }}^{-1}+\frac{1}{T_{2}^{*}}
$$

The relaxation rate $\tau_{\text {relax }}$ is calculated as

$$
\tau_{\text {relax }}^{-1}=2 J_{\text {eff }}(\Delta) \operatorname{coth}\left(\frac{\Delta}{2 k_{B} T}\right) \text {. }
$$

Note that this expression is derived by the weak-coupling approximation and may be inadequate near the resonant point $\left(\Delta=\omega_{0}\right)$. In this Brief Report, we mainly focus on the regime out of the resonant. On the other hand, $1 / T_{2}^{*}$, which is a pure dephasing rate due to a quadratic coupling to the heat bath at the optimal point, is calculated from the coupling strength $r=\lim _{\omega \rightarrow 0} J(\omega) / \omega$ as $^{21}$

$$
\frac{1}{T_{2}^{*}}=\frac{16 \pi}{3}\left(\frac{r}{\Delta}\right)^{2}\left(k_{B} T\right)^{3}=\frac{\pi}{12}\left(\frac{E_{J, \mathrm{eff}}^{2} \gamma}{E_{J, \pi}^{2} E_{C, \pi}}\right)^{2} \frac{\left(k_{B} T\right)^{3}}{\Delta^{2}} .
$$

We estimate the dephasing time in the present flux qubit by using the parameters in Ref. 12. In the experiment, the parameters are chosen as $E_{J} / k_{B}=12 \mathrm{~K}, E_{C} / k_{B}=350 \mathrm{mK}$, $\Delta / k_{B}=160 \mathrm{mK}, T=25 \mathrm{mK}$, and $\alpha=0.8$. There are several candidates of $\pi$ junctions for phase bias. We have estimated dephasing time for several $\pi$-junction systems and found that only underdamped $\pi$ junctions may give a sufficiently long dephasing time.

Here, we discuss underdamped SIFS junctions by using the parameters in Ref. 22; we choose a capacitance and a subgap resistance for unit area as $c=0.08 \mathrm{~F} / \mathrm{m}^{2}$ and $r_{n}=3.0$ $\times 10^{-7} \Omega \mathrm{m}^{2},{ }^{23}$ respectively. In Ref. 22 , the measured critical current density $j_{c}=5.0 \times 10^{4} \mathrm{~A} / \mathrm{m}^{2}$. Here, we take the critical current density as a parameter and discuss its dependence

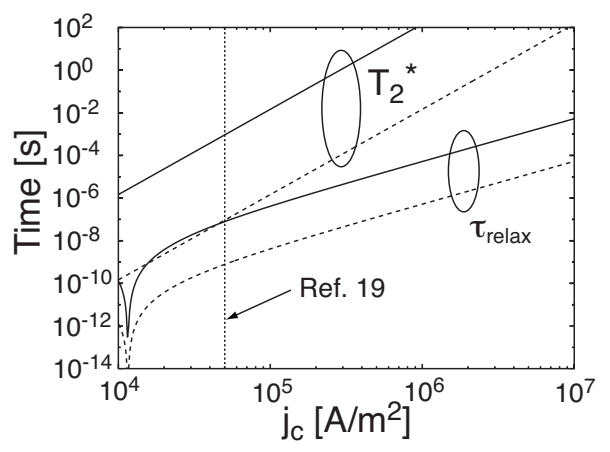

FIG. 2. The estimated relaxation time $\tau_{\text {relax }}$ and pure dephasing time $T_{2}^{*}$. The result for a $10 \times 10 \mu \mathrm{m}^{2}\left(1 \times 1 \mu \mathrm{m}^{2}\right) \pi$ junction is shown by the solid (dashed) line.

keeping $r_{n}$ constant. In Fig. 2, we show the relaxation time $\tau_{\text {relax }}$ and the pure depasing time $T_{2}^{*}$ as a function of the critical current density for $1 \times 1$ and $10 \times 10 \mu \mathrm{m}^{2}$ junctions. In this estimate, the relaxation process is always dominant $\left(\tau_{\text {relax }} \ll T_{2}^{*}\right)$, and therefore the dephasing time $\tau_{\varphi}$ is determined by $\tau_{\text {relax }}$. We find that at the critical current density of Ref. 22, the dephasing time is very short, while long coherence time is obtained for junction with larger area and higher critical current density. The relaxation time has a resonant structure at a low critical current $j_{c}=j_{c}^{*}$, where the resonant condition $\omega_{0}=\Delta$ is satisfied. For $j_{c} \gg j_{c}^{*}$, the relaxation time and the pure dephasing time depend on the junction area $A$ and critical current density $j_{c}$ as $\tau_{\text {relax }} \propto j_{c}^{2} A$ and $T_{2}^{*} \propto j_{c}^{4} A^{2}$, respectively. As seen in Fig. 2, if we use underdamped $\pi$ junctions with large critical current $\left(j_{c} \sim 10^{7} \mathrm{~A} / \mathrm{m}^{2}\right)$ and large junction area $\left(A \sim 10 \times 10 \mu \mathrm{m}^{2}\right)$, coherence time becomes of order of $1 \mathrm{~ms}$, which is sufficiently long compared to the decoherence time limited by other sources. We note that when the relaxation process is dominant, the dephasing time is proportional to $E_{J, \pi}^{2} R$; for realization of long coherence time, we need to increase both the Josephson energy $E_{J, \pi}$ and the subgap resistance $R$ of the $\pi$ junction.

Thus, for long-time coherent operations, one has to improve the quality factor by changing experimental parameters of $\pi$ junctions. Especially important parameter is the critical current density in the $\pi$ state of the junction. In the SIFS junctions described in Ref. 22, the critical current density $j_{c} \simeq 5 \times 10^{4} \mathrm{~A} / \mathrm{m}^{2}$ in the $\pi$ state was still rather low, 3 orders of magnitude less than $j_{c} \simeq 4 \times 10^{7} \mathrm{~A} / \mathrm{m}^{2}$ in a superconductor-insulator-superconductor junction having the same tunnel barrier. Possible reason for the strong suppression of the critical current is the use of diluted alloy $\mathrm{Ni}_{x} \mathrm{Cu}_{1-x}$ which has rather strong disorder leading to fast decay of the supercurrent with increasing ferromagnet $(F)$-layer thickness. Since $0-\pi$ transition occurs at certain critical thickness of the F layer, the supercurrent in the $\pi$ state is much smaller than in the 0 state. However, smallness of $j_{c}$ is not an intrinsic property of SIFS junctions. In a clean homogeneous ferromagnet, the decay length may become much longer than the $0-\pi$ transition thickness. Recent experiments ${ }^{24}$ using $\mathrm{Ni}_{3} \mathrm{Al}$ have demonstrated multiple $0-\pi$ transitions with only modest decay of $\left|j_{c}\right|$ as a function of the thickness of $\mathrm{Ni}_{3} \mathrm{Al}$. Therefore, choosing different materials for a ferromagnet layer 
may finally lead to increasing $j_{c}$ and thus to an increase of the dephasing time of qubits with a SIFS junction.

Finally, we discuss the advantage of the present phase bias. In usual flux qubits, external magnetic flux is needed to produce phase bias along the loop. In many experiments, an external coil with a large current and weak coupling to the qubits has been used. However, this prevents one from using a superconducting shield which provides good shielding of qubits from external flux noise. On the other hand, if one uses a local biasing with a control line, noise in the current source degrades the coherence of the qubit. From Eq. (22), the relaxation time due to this noise can be evaluated as $\tau_{\text {relax }}^{-1}=\left(2 \Delta / \hbar^{2}\right)\left(M I_{p}\right)^{2} / Z$, where $I_{p}=2 \pi E_{J \text {,eff }} / \Phi_{0}$ is a circulating current, $M$ is a mutual inductance, and $Z$ is an impedance of the current source. ${ }^{14,15}$ For obtaining the dephasing time longer than $1 \mathrm{~ms}$, the maximum value of the mutual inductance $M$ is estimated to be $0.03 p H$ for $Z=50 \Omega$. Then, the external current needed for the phase bias becomes $40 \mathrm{~mA}$, which is unrealistically high. Therefore, the use of $\pi$ junctions may be an attractive option for individual phase biasing on qubits.

Recently, another phase biasing scheme with a trapped flux in a superconducting loop has been proposed and demonstrated. ${ }^{25}$ A possible advantage of our scheme using a $\pi$ junction is that we do not need to apply the large external field corresponding to a half flux in the loop ever, either globally or locally. This makes implementation of a superconducting shield simpler.

In summary, we proposed a simple phase bias by $\pi$ junctions for flux qubits and studied dissipation effects at the $\pi$ junction. In the framework of the Caldeira-Leggett model, we derived the effective spectral function of the spin-Boson model and used it for estimate of the dephasing time of the proposed qubit. We showed that for long coherent operation, both the subgap resistance and critical current of the $\pi$ junction have to be increased. We expect that further improvement in quality of $\pi$ junctions enables us to use it for a $\pi$ phase shifter for flux qubits.

We acknowledge H. Hilgenkamp, Ariando, K. Verwijs, A. Andreski, A. V. Ustinov, V. V. Ryazanov, A. K. Feofanov, and S. Kawabata for helpful discussion. This work was supported by the NanoNed Program under Project No. TCS.7029 and was partially supported by CREST, JST.
${ }^{1}$ C. C. Tsuei et al., Phys. Rev. Lett. 73, 593 (1994).

${ }^{2}$ C. C. Tsuei and J. R. Kirtley, Rev. Mod. Phys. 72, 969 (2000).

${ }^{3}$ H. Hilgenkamp et al., Science 422, 50 (2003); H.-J. Smilde et al., Appl. Phys. Lett. 85, 4091 (2004).

${ }^{4}$ J. J. A. Baselmans et al., Nature (London) 397, 43 (1999); J. J. A. Baselmans et al., Phys. Rev. Lett. 89, 207002 (2002).

${ }^{5}$ V. V. Ryazanov et al., Phys. Rev. Lett. 86, 2427 (2001); V. V. Ryazanov et al., Phys. Rev. B 65, 020501(R) (2001); T. Kontos et al., Phys. Rev. Lett. 89, 137007 (2002); H. Sellier et al., Phys. Rev. B 68, 054531 (2003); A. Bauer et al., Phys. Rev. Lett. 92, 217001 (2004); V. A. Oboznov et al., ibid. 96, 197003 (2006).

${ }^{6}$ E. Terzioglu and M. R. Beasley, IEEE Trans. Appl. Supercond. 8, 48 (1998); A. V. Ustinov and V. K. Kaplunenko, J. Appl. Phys. 94, 5405 (2003).

${ }^{7}$ T. Ortlepp et al., Science 319, 1495 (2006).

${ }^{8}$ L. B. Ioffe et al., Nature (London) 398, 679 (1999); G. Blatter, V. B. Geshkenbein, and L. B. Ioffe, Phys. Rev. B 63, 174511 (2001).

${ }^{9}$ Y. Nakamura et al., Nature (London) 398, 786 (1999); T. Yamamoto et al., ibid. 425, 941 (2003); A. Wallraff et al., Phys. Rev. Lett. 95, 060501 (2005).

${ }^{10}$ D. Vion et al., Science 296, 886 (2002); I. Siddiqi et al., Phys. Rev. B 73, 054510 (2006).

${ }^{11}$ J. E. Mooij et al., Science 285, 1036 (1999); C. H. van der Wal et al., ibid. 290, 773 (2000); I. Chiorescu et al., Nature (London) 431, 159 (2004); A. Lupaşcu et al., Nat. Phys. 3, 119 (2007).

${ }^{12}$ I. Chiorescu et al., Science 299, 1869 (2003).

${ }^{13}$ J. M. Martinis et al., Phys. Rev. Lett. 89, 117901 (2002); R. McDermott et al., Science 397, 1299 (2005).

${ }^{14}$ Y. Makhlin et al., Rev. Mod. Phys. 73, 357 (2001).

${ }^{15}$ C. H. van der Wal et al., Eur. Phys. J. B 31, 111 (2003).

${ }^{16}$ M. J. Storcz and F. K. Wilhelm, Appl. Phys. Lett. 83, 2387 (2003).

${ }^{17}$ U. Fano, Phys. Rev. 124, 1866 (1961).

${ }^{18}$ M. Rosenau da Costa et al., Phys. Rev. A 61, 022107 (2000).

${ }^{19}$ F. Bloch, Phys. Rev. 105, 1206 (1957); A. G. Redfield, IBM J. Res. Dev. 1, 19 (1957)

${ }^{20}$ A. J. Leggett et al., Rev. Mod. Phys. 59, 1 (1987).

${ }^{21}$ Y. Makhlin and A. Shnirman, Phys. Rev. Lett. 92, 178301 (2004).

${ }^{22}$ M. Weides et al.Appl. Phys. Lett. 89, 122511 (2006).

${ }^{23} \mathrm{We}$ have estimated the subgap resistance from the McCumber parameter $\beta=2 \pi I_{c} R^{2} C / \Phi_{0}$ measured in Ref. 22 . We have chosen $\beta=10^{6}$ by extrapolating the data of Ref. 22 to the lowtemperature region.

${ }^{24}$ F. Born et al., Phys. Rev. B 74, 140501(R) (2006).

${ }^{25}$ J. B. Majer et al., Appl. Phys. Lett. 80, 3638 (2002); J. H. Plantenberg et al., Nature (London) 447, 836 (2007). 